\title{
Neuropsychiatric Systemic Lupus Erythematosus
}

\author{
Zouhayr Souirti $^{1}$, Mariam Lahlou ${ }^{2}$, Ourda El Ouali ${ }^{1}$, Naima Chtaou ${ }^{1}$, Chadia Aarab $^{3}$, \\ Fatima El Ghazouani ${ }^{3}$, Wafaa Bono ${ }^{2}$, Ismail Rammouz ${ }^{3}$, Faouzi Belahsen ${ }^{1}$, Ouafae Messouak ${ }^{1}$ \\ ${ }^{1}$ Neurology Department, UH Hassan II, Fez, Morocco; ${ }^{2}$ Internal Medicine Department, UH Hassan II, Fez, Morocco; ${ }^{3}$ Psychiatric \\ Department, UH Hassan II, Fez, Morocco. \\ Email: zouhayrsouirti@gmail.com
}

Received June $17^{\text {th }}, 2012$; revised July $25^{\text {th }}, 2012$; accepted August $3^{\text {rd }}, 2012$

Copyright (C) 2013 Zouhayr Souirti et al. This is an open access article distributed under the Creative Commons Attribution License, which permits unrestricted use, distribution, and reproduction in any medium, provided the original work is properly cited.

\begin{abstract}
Systemic lupus erythematosus (SLE) is a chronic autoimmune disease characterized by multisystemic involvement and diverse manifestations. Neuropsychiatric systemic lupus erythematosus (NPSLE) is a complex neurological disorder characterized by neuropsychological dysfunction. NPSLE is associated with increased morbidity and mortality. In 1999, the American College of Rheumatology developed 19 discrete neuropsychiatric syndromes that comprised NPSLE. Nervous system disease in systemic lupus erythematosus is manifested by a wide variety of clinical manifestations. The pathogenesis of NPSLE is due to autoantibodies, neuronal and non neuronal antigens and the generation of proinflammatory cytokines and mediators. Anatomopathological lesions are attributed to in situ thrombosis, edema, hemorrhage, vasculitis, atherosclerosis or atheroembolism. The diagnosis of NPSLE remains largely one of exclusion and is approached by clinical evaluation, and supported when necessary by autoantibody profiles, diagnostic imaging, electrophysiologic studies and objective assessment of cognitive performance. Brain MRI abnormalities in NPSLE might show small punctate focal lesions in white matter being the most common MRI finding, followed by cortical atrophy, ventricular dilation, cerebral edema, diffuse white matter abnormalities, focal atrophy, cerebral infarction, acute leukoencephalopathy and intracranial hemorrhage. The treatment is based on the use of symptomatic therapies, immunosuppressives and non-pharmacologic interventions. This review paper was designed to understand the pathophysiology for better management of NPSLE.
\end{abstract}

Keywords: Central Nervous System; Systemic Lupus Erythematosus; MRI

\section{Introduction}

Systemic lupus erythematosus (SLE) is an autoimmune multifactorial disease. It may affect every organ in the body without any specificity. It is characterized by a relapsing-remitting course. Biologically, the disease is characterized by the production of multiple auto-antibodies against nuclear components. Some of these antibodies, such as anti-dsDNA (or anti-Sm) are characteristic and specific to the affection. Neurological involvement in SLE, commonly called neuropsychiatric lupus (NPSLE), is the third leading cause of death. Its prevalence is variously estimated according to the established criteria. Clinically, the disease sometimes starts with neurological manifestations. There is currently an improvement in pathophysiology comprehension, but the treatment of NPSLE is still a great challenge. It is based on several therapeutic molecules such as immunosuppressive and immunomodulatory therapies.

\section{Pathogenesis}

\subsection{Vasculopathy}

Pathological studies have shown that neurological involvement is mainly microvascular. It is observed in $65 \%$ of cases of cerebral lupus and affects mainly arterioles of a diameter less than $100 \mu \mathrm{m}$. It rarely takes the aspect of vasculitis with inflammatory cell infiltration of vessels wall tunics $(7 \%)$. The anomalies are rather proliferative and degenerative. There are often multiple foci of microinfarcts and microhemorrhages.

\subsection{Auto-Antibodies (Table 1)}

About half of patients suffering from lupus will be affected by neuropsychiatric manifestations in the course of their disease. The etiology of the neuronal impairment associated with these manifestations is still unclear and probably multiple. The responsibility of auto-antibodies 
Table 1. Autoantibodies associated with cognitive impairment and other neuropsychiatric manifestations of SLE.

\begin{tabular}{l} 
Autoantibodies Associated with Cognitive Impairment in SLE \\
Anti-neuronal antibodies \\
Anti-phospholipid antibodies \\
Anti-NR2 receptor antibody \\
Lymphocytotoxic antibody \\
\hline Autoantibodies Associated with Other NPSLE Manifestations \\
Anti-ribosomal P antibody \\
Anti-neurofilament antibody \\
Anti-endothelial cell antibody \\
Anti-Ro antibody \\
Anti-Smith antibody \\
Reference: [23].
\end{tabular}

is suggested for some of these symptoms. The description of pathogenic auto-antibodies against dsDNA and the subunits NR2A and NR2B of the ionotropic receptor activated by glutamate (NMDA-R) illustrates this hypothesis. Early studies have shown that $40 \%$ of lupus patients have an NR2A/B anti-epitope title, but the presence of these serum antibodies is not always associated with the observation of neurological or neuropsychiatric disorders. However, cerebrospinal fluid (CSF) of more than half of NPSLE patients contains these autoantibodies. Their presence in the serum precedes their passage into the CSF where their harmful effects are observed. This passage depends on the accidental opening of the blood brain barrier (BBB). In conclusion, detection of epitope NR2A/B serum autoantibodies is an indicator of the potential occurrence of neuropsychiatric manifestations.

Autoimmune antiphospholipid antibodies, against phospholipid binding proteins such as b2-glycoprotein I and prothrombin, are associated with predominately focal manifestations of NPSLE. The most common neurologic disorders are those of vascular origin, such as transient cerebral ischemia or stroke, but other associations include seizures, chorea, transverse myelitis and cognitive dysfunction. The pathogenic mechanism for this subset of autoantibodies in NPSLE is thrombosis within vessels of different caliber and subsequent cerebral ischemia. A procoagulant state can be induced through acquired resistance to proteins $\mathrm{C}$ and $\mathrm{S}$, platelet aggregation and direct activation of endothelial cells. However, the intrathecal production of antiphospholipid antibodies in patients with NPSLE, their association with diffuse cognitive impairment and in vitro evidence indicating modulation of neuronal cell function raise the possibility of an alternative pathogenic mechanism [1].

\section{Diagnosis}

\subsection{SLE Diagnosis}

The diagnosis of lupus is based mainly on clinical exami- nation supported by laboratory data. At an early stage of the disease, a number of general symptoms may mislead the diagnosis. These symptoms can include fatigue, weight loss and a prolonged fever. These signs often constitute the initial complaints and patients often attribute them to other conditions than lupus.

Over time, various but more specific manifestations will appear affecting different organs either successively or in combination. The most frequently encountered initial manifestations are inflammatory arthralgias or an actual inflammatory arthritis. After several years, the clinical manifestations are diverse, and all organs may be affected.

According to the new criteria, SLE is considered present if a patient has simultaneously or successively any 4 or more of the criteria below:

1) Butterfly-shaped Facial rash;

2) Discoid rash;

3) Photosensitivity;

4) Oral or nasopharyngeal ulcers;

5) Non-deforming arthritis of one or more peripheral joints with pain on mobilization, tenderness, effusion or swelling of the soft parts without joint deformation;

6) Pleuritis or pericarditis (serositis);

7) Persistent proteinuria $>0.5 \mathrm{~g} / 24$ hours;

8) Neurologic disorders: Psychosis or convulsions (in the absence of drug intake and/or metabolic cause);

9) Hematologic disorder: Leucopenia $<4000$ detected on two occasions, thrombocytopenia $<100,000$ (in absence of drugs known to induce it), lymphopenia $<1500$ detected on two occasions, hemolytic anemia;

10) Immunological abnormalities: Presence of antibodies to DNA and to Sm at significant rates, presence of anti-phospholipid antibodies (dissociated positive syphilis serology controlled twice with an interval of 6 months, positive anticardiolipin IgG and/or IgM).

It should be noted that these are classification criteria and not diagnostic criteria. Their use as a diagnostic tool makes them lose their sensitivity essentially at the time of diagnosis. Indeed, all the criteria are not necessarily present at this time. It may take many years for the fourth criterion to appear and help classify "correctly" the patient. At present, some authors developed an approach based on the use of weighted scores: some clinical or immunological factors have "more weight" than others for the diagnosis of SLE. By assigning each element a mathematical value, we can obtain a score for each patient and thus provide a diagnostic score.

\subsection{NPSLE Diagnosis}

The first step in the management of a patient with SLE and presenting with a neuropsychiatric event is to determine whether the event can be convincingly attributed to 
SLE, a complication of the disease or its therapy, or whether it reflects a coincidental disease (Figure 1). This is achieved largely by a process of exclusion, given the absence of a diagnostic gold standard for most of the neuropsychiatric manifestations that occur in SLE. The diagnosis is then based on a careful analysis of the clinical, laboratory and imaging data on a case-by-case basis.

\section{Clinical Manifestations of NPSLE}

\subsection{Neurological Manifestations}

The most common neurological manifestations in SLE are central nervous system syndromes (35\% to $75 \%$ of published series). They can be diffuse or focal, acute or chronic. We must always differentiate them from focal impairments resulting from an ischemic stroke or from diffuse impairment related to metabolic or electrolytic disturbances (renal failure), side effects of medications including steroids and opportunistic infections [2].

Epileptic seizures (14\% to 25\%) usually present with generalized tonic-clonic, more rarely with simple partial or complex motor or sensory seizures [3].

Epileptic seizures are statistically associated with the presence of antiphospholipid antibodies [4].

This association could be elicited by two distinct mechanisms: an epileptogenic scar of a cerebral infarction caused by antiphospholipid antibodies (APL) or direct interaction of APL with the brain parenchyma. Seizures in SLE patients could be secondary to hypokalemia, chronic renal failure, malignant hypertension and toxicity of corticosteroids or antimalarials.

Meningeal and meningoencephalitic syndromes can be directly linked to lupus, but this diagnosis requires ex- cluding infection or a drug induced syndrom as it can happen with Ibuprofen and immunoglobulin infusions.

Headaches are rather a common complaint that can be associated with the disease itself. They are caused by stress. Most common types of headaches are migraine without aura, migraine with aura, tension headache and idiopathic intracranial hypertension. According to Mitsikostas et al. [5], in a very recent study, headache is related to active lupus, especially in patients with active systemic disease; however, no available data have established that headaches may be induced by lupus.

Patients with SLE are at increased risk of stroke. Cerebro-vascular disease affects between $2 \%$ and $15 \%$ of patients with SLE. Acute ischemic stroke is the most common form. After a cerebro-vascular event, the risk of recurrence is very high. Causes of stroke in SLE include thrombosis (role of antiphospholipid antibodies), hemorrhage, high blood pressure and thrombocytopenia [6]. Recurrent ischemic stroke should be investigated to look for an embolic cause originating from a verrucous endocarditis.

Movement disorders are rare (1\% - 3\% of patients) and chorea is the most common expression [7]. Both chorea and transverse myelitis are associated with the presence of antiphospholipid antibodies [8]. If arterial thrombosis is considered a probable cause of transverse myelitis, etiology of chorea is less clear. Some speculations point the direct interaction of antiphospholipid antibodies with neuronal structures of the basal ganglia [8].

Transverse myelitis, characterized by a sudden paraplegia, has a poor outcome. Patients presenting a transverse myelitis may develop an optic neuropathy. Progressive multifocal leukoencephalopathy is a progressive

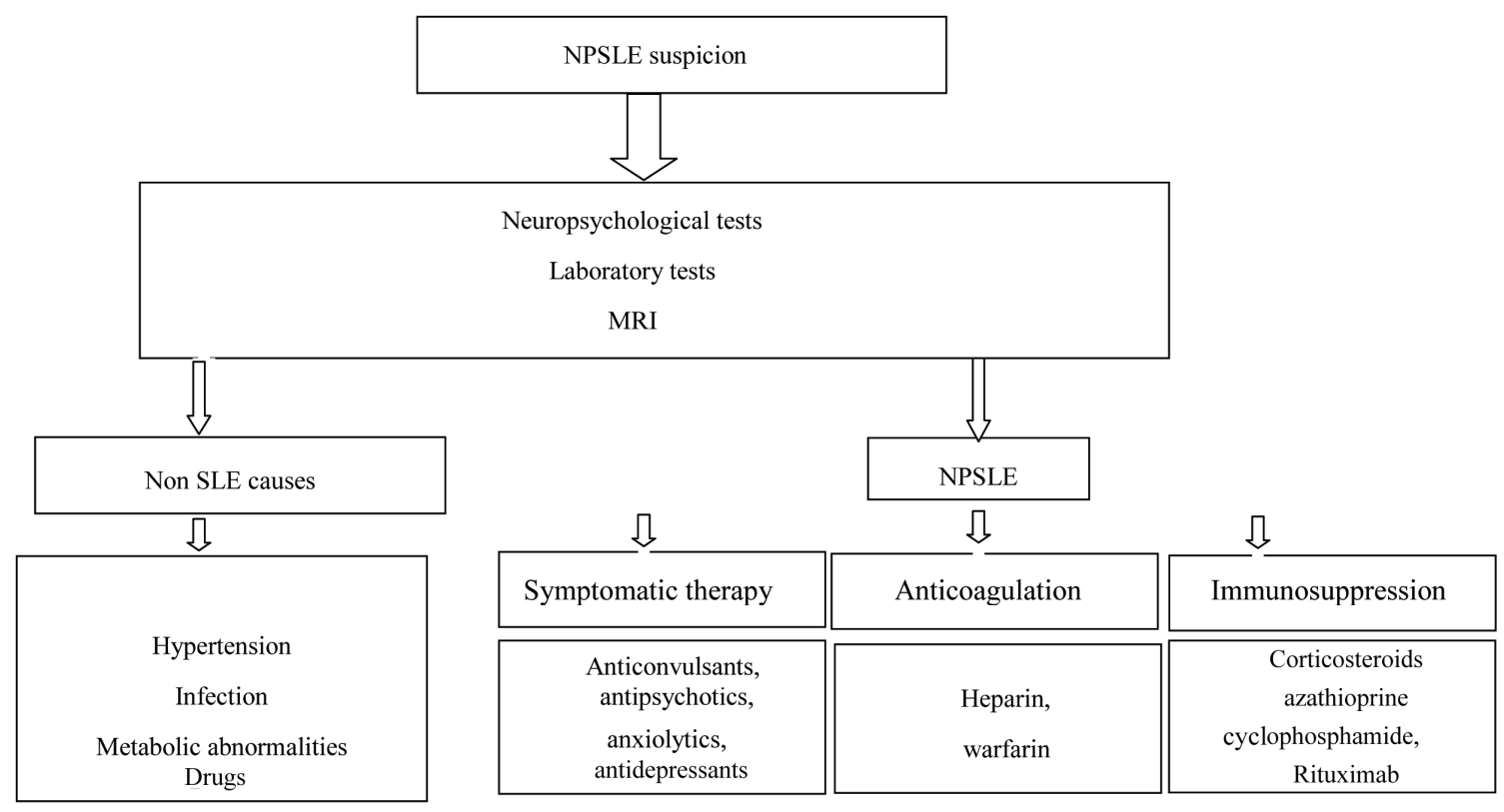

Figure 1. Diagram of NPSLE management. 
disease that was reported to occur after immunosuppressive therapies [9].

Cranial and peripheral nerves impairment affects $10 \%-15 \%$ of patients and usually occurs during relapses. They are transient and respond to conventional therapy (high doses of glucocorticoids) [10]. Cranial nerves impairment results in optic nerve damage, impairment of extraocular muscles, trigeminal neuralgia, facial paralysis, velum paralysis, deafness, nystagmus and vertigo. Peripheral neuropathy, much rarer than necrotizing vasculitis, can be presented in three main aspects: distal sensory neuropathy, mononeuropathy or multiple mononeuropathy or acute polyradiculoneuropathy.

\subsection{Cognitive and Psychiatric Disorders of Lupus}

The issue of psychiatric disorders in SLE is more complex. It could be a "simple" co-morbidity with coexisting disorders but without any causal relationship with SLE, or it could be a psychiatric disorder with a direct causal relationship with SLE. In this last condition, psychiatric disorders may be the consequence of central nervous system impairment or they could be iatrogenic related to corticosteroids.

Globally, the wide range of frequency of neuropsychiatric manifestations reported in the literature is striking, with proportions ranging from $14 \%$ to $75 \%$ depending on the used criteria [11]. $16 \%-52 \%$ of depressive disorders and $5 \%$ to $11 \%$ of psychotic disorders are reported. For these proportions, authors didn't distinguish between comorbid, reactionnal, iatrogenic psychiatric disorders and disorders directly related to SLE.

These widely varying proportions have several reasons: duration of patient monitoring, diagnostic criteria, but also difficulties to confirm whether the neuropsychiatric disorder is directly attributable to SLE or is caused by treatment or secondary to complications of the disease.

\subsubsection{Cognitive Disorders}

Neurocognitive impairment is one of the most common and clinically challenging of all SLE manifestations. However, its pathophysiology remains poorly understood. Their diagnosis requires administration of an extensive neuropsychological testing battery (Table 2). Dementia can inaugurate lupus or occur late in the disease course [12].

\subsubsection{Primary Psychiatric Disorders}

Psychotic disorders: They are often presented as confusional psychosis combining agitation and perceptual disturbances. They are present in 5\% - 15\% of cases. Some patients may present with atypical clinical syndromes: sub-delusional state with persecutory themes mainly. Acute psychotic manifestations often occur early in the
Table 2. One-hour neuropsychological battery proposed by the ACR to assess cognitive function in adults with SLE.

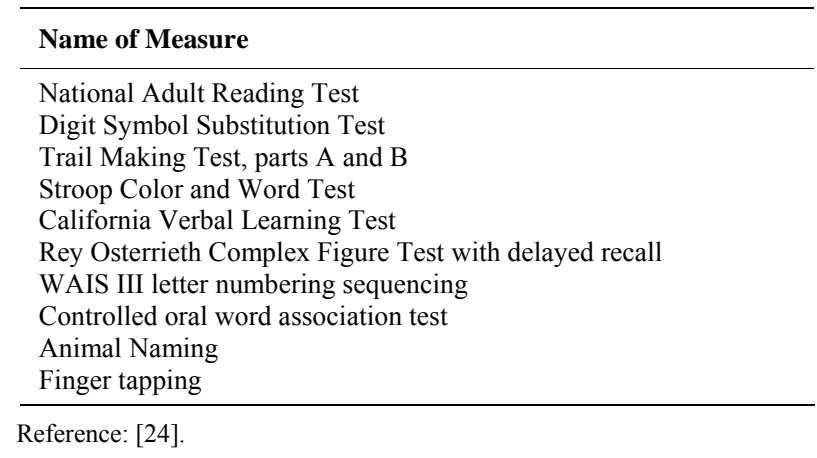

course of lupus.

Mood and adjustment disorders: The depressive episode may inaugurate the illness in some patients but it might be also reactionary to the diagnosis announcement or occur during the course of the disease as a response to the stress of the chronic disease and the serious social impacts.

Anxiety disorders: The manifestations of anxiety are common such as panic attacks or phobic and obsessive disorders. In some cases, the disorders are pre-existing and exacerbated because of SLE.

The main distinguishing features are the chronology of troubles onset, the presence of other physical impairments, circumstances of onset and a change in the usual psychiatric symptomatology in a patient with a comorbid depressive disorder [13]. Sixty-one percent of lupus-related disorders occur in the first year against $57 \%$ in the first 15 days after treatment with corticosteroids.

Disorders due to NPSLE often occur when doses of corticosteroids are decreased. Psychotic disorders are due to NPSLE rather than to corticosteroids. In $92 \%$ of cases, any decrease in corticosteroids doses can relieve symptoms [14].

\section{Imaging}

In their study, Oseph et al. (2007) indicated that the brain computed tomography scan (CT scan) was abnormal in only $35 \%$ of cases, whereas Magnetic Resonance Imaging (MRI) was abnormal in $65 \%$ of cases. The brain CT scan is still useful to identify cerebral hemorrhage and large infarction [15], but MRI assessing is much more sensitive to subtle ischemia and cerebritis. Ischemic type lesions are the most common [2].

MRI shows abnormalities in $15 \%-50 \%$ of asymptomatic patients [16]. Lesions are often clinically silent as in other white matter diseases such as multiple sclerosis where MRI is 5 to 10 times more sensitive than clinical examination [17].

$\mathrm{T} 2$ images are more sensitive than T1 images to detect abnormalities in patients with lupus. MRI T2 images 
identify pathological processes, responsible for edema. Focal neurological lesions are located mainly in the periventricular region and subcortical white matter, usually in the territory of a major cerebral blood vessel [18]. How- ever, these white matter lesions are not specific and are most frequently attributed to hypertension, duration of disease and small vessel disease related to age more than lupus.

Positron Emission Tomography and Single Photon Emission Tomography (SPECT) can identify early biochemical and blood flow abnormalities in patients with lupus. Castellino et al (2008) reported that the combination of SPECT and MRI appears more useful and may help clinicians evaluate NPSLE patients [2]

\section{Therapeutic Management}

The treatment of NPSLE has changed considerably with the outstanding improvement of pathophysiological mechanisms. The treatment uses immunosuppressive and immunomodulatory therapies and plasma exchange. Opinions concerning treatment are divergent. At present, there are available data based on retrospective studies, clinical observations and personal experiences.

The choice of immunosuppressive therapy depends on the pathogenic mechanisms and the severity of the clinical presentation [19].

Several studies have shown the benefit of cyclophosphamide over corticosteroids [20]. We report the experience of the department Belgrade University Clinical Center where 60 NPSLE patients were studied. Two patient groups were compared. The first group received a monthly bolus of cyclophosphamide at a dose ranging between 200 and $400 \mathrm{mg} / \mathrm{m}^{2}$ associated to oral corticosteroids at $20 \mathrm{mg}$. The second group received oral corticosteroids at the same dose as the first group associated with a synthetic antimalarial. The results showed statistically significant superiority of cyclophosphamide [20].

Use of intrathecal bolus of Dexamethasone associated with Methotrexate is reported in some series with good clinical results [19]. The use of plasmapheresis is recommended when conventional treatment (corticosteroids associated with immunosuppressive therapy) fails. Experience has shown that plasma exchange in patients refracttory to conventional therapy induced a complete remission in 7 of 13 patients [21]. Anticoagulation is recommended in thrombotic processes, particularly the antiphospholipid syndrome.

Lupus is an autoimmune disease characterized by the presence of lesions induced by $\mathrm{T}$ cells self-activation and production of anti-B cells auto-antibodies. This mechanism suggested the idea to propose Rituximab for the treatment of resistant NPSE [22].

10 patients were included in the "Rituximab" study. These patients were diagnosed NPSLE patients accord- ing to the ACR criteria, with an active neurological disease resistant to conventional therapy. The treatment protocol consisted of using either Rituximab at a dose of $375 \mathrm{mg} / \mathrm{m}^{2}$ weekly for 2 weeks or $500 \mathrm{mg}$ weekly for 4 weeks. The study showed that patients treated with Rituximab were stabilized with a complete clinical, radiological and biological remission [22]. We can deduce that there is no codified therapeutic strategy for NPSLE. This requires prospective studies and laboratory confirmation.

\section{Conclusion}

The most prevalent manifestations of neuropsychiatric lupus erythematosus are headache, mood disorders and cognitive dysfunction. Neuropsychiatric events in SLE patients may be attributable to lupus or non-SLE causes. The diagnosis of NPSLE is largely one of exclusion; it is important to rule out non-SLE causes. The treatment of NPSLE is individualized and consists of symptomatic therapies, anticoagulation in antiphospholipid syndrome, corticosteroids and immunosuppression.

\section{Acknowledgements}

To EL HACHIMI Mohamed, for English text proofreading.

\section{REFERENCES}

[1] J. G. Hanly and M. J. Harrison, "Management of Neuropsychiatric Lupus," Best Practice \& Research Clinical Rheumatology, Vol. 19, No. 5, 2005, pp. 799-821. doi:10.1016/j.berh.2005.04.003

[2] T. S. Ramachandran, "Neurologic Manifestations of Systemic Lupus," 2011. http://emedicine.medscape.com/article/1146456-overview

[3] J. Mikdashi, A. Krumholz and B. Handwerger, "Factors at Diagnosis Predict Subsequent Occurrence of Seizures in Systemic Lupus Erythematosus," Neurology, Vol. 64, No. 12, 2005, pp. 2102-2107. doi:10.1212/01.WNL.0000165959.98370.D5

[4] M. J. Cuadrado and M. A. Khamashta, "Can Neurologic Manifestation of Hughes (Antiphospholipide Syndrome Be Distinguished from Multiple Sclerosis) Analyse of 27 Patients and Review of Literature," Medicine, Vol. 79, No. 1, 2000, pp. 57-68. doi:10.1097/00005792-200001000-00006

[5] D. D. Mitsikostas, P. P. Sfikakis and P. J. Goadsby, "A Meta-Analysis for Headache in Systemic Lupus Erythematosus: The Evidence and the Myth," Brain, Vol. 127, No. 5, 2004, pp. 1200-1209. doi:10.1093/brain/awh146

[6] H. Ainiala, J. Loukkola, J. Peltola, M. Korpela and A. Hietaharju, "The Prevalence of Neuropsychiatric Syndromes in Systemic Lupus Erythematosus," Neurology, Vol. 57, No. 3, 2001, pp. 496-500. doi:10.1212/WNL.57.3.496

[7] H. Ainiala, A. Hietaharju, J. Loukkola, J. Peltola, M. 
Korpela and R. Metsanoja, "Validity of the New American College of Rheumatology Criteria for Neuropsychiatric Lupus Syndromes: A Population-Based Evaluation," Arthritis Rheum, Vol. 45, No. 5, 2001, pp. 419-423. doi:10.1002/1529-0131(200110)45:5<419::AID-ART360 $\geq 3.0 . \mathrm{CO} ; 2-\mathrm{X}$

[8] R. Cervera, R. A. Asherson, J. Font, M. Tikly, L. Pallares and A. Chamorro, "Chorea in the Antiphospholipid Syndrome. Clinical, Radiologic, and Immunologic Characteristics of 50 Patients from Our Clinics and the Recent Literature," Medicine, Vol. 76, No. 3, 1997, pp. 203-212. doi:10.1097/00005792-199705000-00006

[9] F. Ahmed, T. Aziz and L. D. Kaufman, "Progressive Multifocal Leukoencephalopathy in a Patient with Systemic Lupus Erythematosus," The Journal of Rheumatology, Vol. 26, No. 7, 1999, pp. 1609-1612.

[10] L. Barile and C. Lavalle, "Transverse Myelitis in Systemic Lupus Erythematosus-The Effect of IV Pulse Methylprednisolone and Cyclophosphamide," Journal of Rheumatology, Vol. 19, No. 3, 1992, pp. 370-372.

[11] J. G. Hanly, K. Cassell and J. D. Kiska, "Cognitive Function in Systemic Lupus Erythematosus: Results of a FiveYear Prospective Study," Arthritis \& Rheumatism, Vol. 40, No. 8, 1997, pp. 1542-1543. doi:10.1002/art.1780400825

[12] R. M. Carbotte, S. D. Denburg and J. A. Denburg, "Cognitive Dysfunction and Systemic Lupus Erythematosus," In: R. G. Lahit, Ed., Systemic Lupus Erythematosus, Churchill Livingstone, New York, 1992, pp. 865-881.

[13] J. C. Piette, D. Marra, C. Lafitte and Z. Amoura, "Neurological Manifestations of Systemic Lupus Erythematosus," In: H. Dechy, B. Wechsler, P. Hausfater, G. Rancurel and J. C. Piette, Eds., Neurological Damage during Systemic Diseases, Flammarion Medicine-Sciences, Paris, 2003, pp. 141-147.

[14] O. Meyer and M. F. Kahn, "Lupus Erythematosus," In: M. F. Kahn, A. P. Peltier, O. Meyer and J. C. Piette, Eds., Systemic Diseases and Syndromes, Flammarion MedicineSciences, Paris, 2000, pp. 131-368.

[15] F. G. Joseph, G. A. Lammie and N. J. Scolding, "CNS Lupus: A Study of 41 Patients," Neurology, Vol. 69, No. 7, 2007, pp. 644-654. doi:10.1212/01.wnl.0000267320.48939.d0

[16] M. J. Jarek, S. G. West, M. R. Baker and K. M. Rak,
"Magnetic Resonance Imaging in Systemic Lupus Erythematosus Patients without a History of Neuropsychiatric Lupus Erythematosus," Arthritis \& Rheumatism, Vol. 37, No. 11, 1994, 1609-1613. doi:10.1002/art.1780371108

[17] D. W. Paty, "Magnetic Resonance Imaging in the Assessment of Disease Activity in Multiple Sclerosis," Canadian Journal of Neurological Sciences, Vol. 15, No. 3, 1988, pp. 266-272.

[18] C. L. Bell, C. Partington, M. Robbins, F. Graziano, P. Turski and S. Kornguth, "Magnetic Resonance Imaging of Central Nervous System Lesions in Patients with Lupus Erythematosus. Correlation with Clinical Remission and Antineurofilament and Anticardiolipin Antibody Titers," Arthritis \& Rheumatism, Vol. 34, No. 4, 1991, pp. 432-441. doi:10.1002/art.1780340408

[19] G. Sanna, L. Bertolaccini and A. Mathieu, "Central Nervous System Lupus: A Clinical Approach to Therapy," Lupus, Vol. 12, No. 12, 2003, pp. 935-942.

[20] L. Stojanovich, R. Stojanovich, V. Kostich and E. Dzjolich, "Neuropsychiartic Lupus Response Favourable to Low Dose i.v. Cyclophosphamide and Prednisolone," Lupus, Vol. 12, No. 1, 2003, pp. 3-7. doi:10.1191/09612033031u251oa

[21] P. Bartolucci, S. Bréchignac, P. Cohen, V. Le Guern and L. Guillevin, "Adjunctive Plasma to Treat Neuropsychiatric Lupus Exchanges: A Retrospective Study Were $10 \mathrm{~Pa}-$ tients," Lupus, Vol. 16, No. 10, 2007, pp. 817-822. doi:10.1177/0961203307081840

[22] T. Mikiko, S. Kazuyoshi, K. Daisuke, I. Yoshitaka, T. Fujii, N. Shingo, T. Shizuyo, N. Masao, I. Shigeru, A. Taeko, M. Tsuneyo and T. Yoshiya, "Efficacy of Rituximab (Anti-CD20) for Refractory Systemic Lupus Erythematosus Involving the Central Nervous System," Annals of the Rheumatic Disease, Vol. 66, No. 4, 2007, pp. 470-475.

[23] G. Zandman-Goddard, J. Chapman and Y. Shoenfeld. "Autoantibodies Involved in Neuropsychiatric SLE and Antiphospholipid Syndrome," Seminars in Arthritis and Rheumatism, Vol. 36, No. 5, 2007, pp. 297-315.

[24] J. A. Mikdashi, J. M. Esdaile, G. S. Alarcón, et al., “Ad Hoc Committee on Lupus Response Criteria: Cognition Sub-Committee," Lupus, Vol. 16, No. 6, 2007, pp. 418425. 